\title{
Research of Discharge and Generation of Fast Electrons in Atmospheric Pressure Inhomogeneous Gas Media
}

\author{
V.V. Lisenkov*, V.V. Osipov, D.S. Mastyugin and V.I. Solomonov \\ Institute of Electrophysics UD RAS, Amundsena st., 106, Ekaterinburg, 620016, Russia
}

\begin{abstract}
The paper presents the results of our theoretical investigations of fast electron beam generation in high-temperature plasma of a laser plume and further acceleration of electrons in atmosphere. The discharge formation in these inhomogeneous media was also investigated. Results of calculations have shown an opportunity of generation of fast beam with a current in a range $\approx 10^{2}-10^{3}$ A from the laser plume with the length of $5 \mathrm{~mm}$ and the area of $0.1 \mathrm{~mm}^{2}$. The main reason of limitation of fast electrons current pulse was the breakdown of the discharge gap. The preliminary experiments carried out by us have shown the correctness of chosen direction.
\end{abstract}

PACS numbers: 41.75.Lx, 52.80.Tn

\section{Introduction}

As is known, the effect of fast electrons generation in high-pressure gas media was discovered more than 30 years ago. However, for these days it has not found a serious application because of low efficiency of fast electrons output in atmospheric air.

The current state of this problem is described in detail in review papers $[1,2]$. Here we should note that the basic problem is a requirement of high $E / N(E$ - electric field strength, $N$ - number density of gas molecules) applying for transition of noticeable part of electrons into continuous acceleration (runaway or escape) mode. The required high values of $E / N\left(\approx 10^{5}-10^{6} \mathrm{~V} /(\mathrm{cm}\right.$ atm $)$ or $\left.10^{3}-10^{4} \mathrm{Td}\right)$ could be realized only in areas of electric field amplification, for example, in front of a spark channel, near a cathode of special shape, etc., at mean electric fields $<10^{3} \mathrm{Td}$.

The characteristic time of breakdown of a discharge gap in such conditions lays in nanosecond and sub nanosecond range and decreases with growth of $E$. Hence, a voltage rise time $\left(\tau_{\mathrm{f}}\right)$ should not exceed a value $\approx 10^{-10}-10^{-9} \mathrm{~s}$.

Thus, a traditional way to solve the problem is to increase of $E$ and decrease of $\tau_{\mathrm{f}}$, as necessary requirement.

Taking into account that the part of escaping electrons is the function of $E / N$, we suggest to increase this parameter not by increasing $E$, but with a help of decreasing $N$, by making high-temperature area near the cathode.

Such area can be, for example, a laser plume or a spark channel, which finished their thermal expansion.

\section{Theory}

For the theoretical analysis of the suggested approach we worked out the one-dimensional numerical model consisting of the system of balance equations for slow and

\footnotetext{
* corresponding author; e-mail: lisenkov@iep.uran.ru
}

fast electrons, ions, and Poisson equation

$$
\begin{aligned}
& \frac{\partial n_{\mathrm{e}}}{\partial t}+\frac{\partial n_{\mathrm{e}} \mu_{\mathrm{e}} E}{\partial x}=\nu_{\mathrm{i}}(E) n_{\mathrm{e}} \\
& \quad+\int_{\varepsilon_{\mathrm{th}}}^{\infty} v n_{\mathrm{fe}}(\varepsilon, x) \sigma_{\mathrm{i}}(\varepsilon) \mathrm{d} \varepsilon-\nu_{\mathrm{esc}}(E) n_{\mathrm{e}}-k r n_{\mathrm{e}} n_{\mathrm{i}}, \\
& \frac{\partial n_{\mathrm{i}}}{\partial t}-\frac{\partial n_{\mathrm{i}} \mu_{\mathrm{i}} E}{\partial x}=\nu_{\mathrm{i}}(E) n_{\mathrm{e}} \\
& \quad+\int_{\varepsilon_{\mathrm{th}}}^{\infty} v n_{\mathrm{fe}}(\varepsilon, x) \sigma_{\mathrm{i}}(\varepsilon) \mathrm{d} \varepsilon-k r n_{\mathrm{e}} n_{\mathrm{i}} \\
& \frac{\partial^{2} \varphi}{\partial x^{2}}=\frac{e}{\varepsilon_{\mathrm{o}}}\left(n_{\mathrm{e}}-n_{\mathrm{i}}\right) .
\end{aligned}
$$

$n_{\mathrm{i}}, n_{\mathrm{e}}, n_{\mathrm{fe}}-$ concentration of ions, slow and fast electrons respectively, $\nu_{\mathrm{i}}$ - ionization rate by slow electrons, $\nu_{\text {esc }}=1 / \tau_{\text {esc }}, \tau_{\text {esc }}(E)$ - time of transition of electron into escape (or runaway), $\mu_{\mathrm{e}}, \mu_{\mathrm{i}}$ - mobilities of electrons and ions, $k r$ - recombination coefficient, $\varepsilon_{\text {th }}$ threshold energy for electrons to be in runaway mode.

The kinetic coefficients as function of electric field, including the probability of transition of electrons into escape mode, were received as a result of modelling of electron motion by Monte Carlo method. We did not include $n_{\mathrm{fe}}$ in Poisson equation, because $n_{\mathrm{fe}}$ was smaller by 2 order than $n_{\mathrm{e}}$ and $n_{\mathrm{i}}$.

Energy and coordinate distribution of fast electrons $n_{\mathrm{fe}}(\varepsilon, x)$ was calculated as follows:

$$
n_{\mathrm{fe}}\left(\varepsilon_{i}, x_{j}, t+\Delta t\right)=\sum_{k, m} p\left(\varepsilon_{k, i}, x_{m, j}\right) n_{\mathrm{fe}}\left(\varepsilon_{k}, x_{m}, t\right) .
$$

$p\left(\varepsilon_{k, i}, x_{m, j}\right)$ - probability of occurrence of electron with energy $\varepsilon_{k}$ and coordinate $x_{m}$ within the cell $x_{j} \varepsilon_{I}$ during the time interval $\Delta t ; i, j$ and $k, m$ - numbers of coordinate-energy cells. Meanings of $p\left(\varepsilon_{k, i}, x_{m, j}\right)$ were received as a result of modeling by Monte Carlo method.

We made test calculations for the conditions of experiment which was carried out recently in our institute with 
use of electron accelerator RADAN and the cathode as a thin-walled tube [3].

To avoid the solution of a two-dimensional problem, but at the same time to take into account the amplification of electric field near the tube cathode we took the results of numerical solution of the two-dimensional Laplace equation obtained by Yalandin with the help of computer code SAM [3]. The received amplification factor of electric field near the cathode was used in our one-dimensional calculations.

\section{Results and discussion}

The calculated oscillograms of the fast electron current are put as dotted curves on experimental oscillogram from paper [3] (Fig. 1). The oscillograms with peak values of $2.8 \mathrm{~A}$ and $0.5 \mathrm{~A}$ were calculated for cases of presence and absence of 1 ns pre-pulse (for details see [3]). They are in qualitative agreement with the experimental results. In the case of pre-pulse presence the peak current obtained in experiment was $1.2 \mathrm{~A}$, and in the case of pre-pulse absence the current of fast electrons was not obtained.

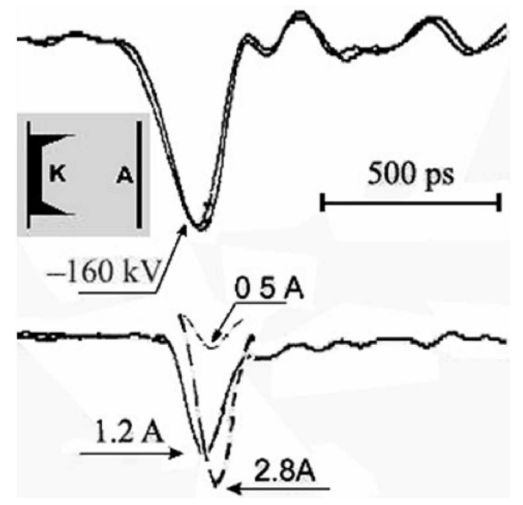

Fig. 1. Experimental oscillograms of voltage on the gap and a current of fast electrons (solid curves) [3], and calculated oscillograms of fast electron current (dash curves). Geometry of problem is in the inset.

According to our calculations, in this experiment the fast electron current was limited by small volume of area, where the conditions for runaway mode were realized. The area of generation of fast electrons can be essentially enlarged with a help of heated area of laser plume.

For modelling of fast electron beam generation in heated area we solved the following problem (Fig. 2a). The discharge gap consisted of two areas. Area near cathode was the laser plume (marked by grey colour) that was produced by laser radiation pulse from the graphite cathode. The plume had following parameters: length $0.5 \mathrm{~cm}$, area of $0.1 \mathrm{~cm}^{2}$ temperature - equal to temperature of graphite sublimation $(4500 \mathrm{~K})$, concentration of neutral particles $N<0.1 N_{\mathrm{L}}\left(N_{\mathrm{L}}\right.$ - Loschmidt number). Such parameters can be easily received with the help of pulsed $\mathrm{CO}_{2}$ laser [4]. Between the laser plume and anode was the air gap $\left(N=N_{\mathrm{L}}\right)$ with varied length. For simplicity we supposed that the rate of voltage rising was constant and was provided by properties of a key. The real forming line was conventionally exchanged with a chain, consisting of the power supply and resistor, which was equal to impedance of a line $(50 \Omega)$. Such approach is valid, while the wave of line discharge will not reach the edge of a line.

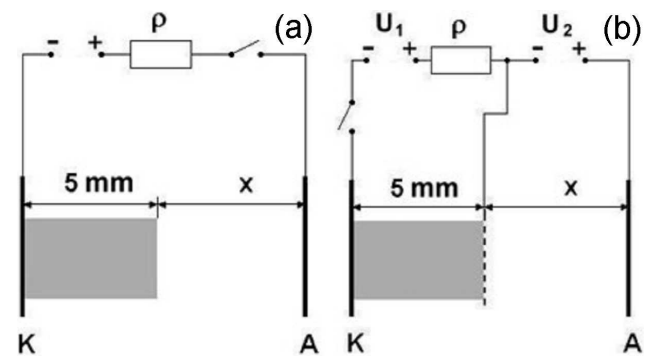

Fig. 2. Illustration of the numerical problem solved. Items (a) and (b) are described in the text.

Calculated oscillograms of a voltage on a discharge gap and currents of plasma and fast electrons are shown in Fig. 3. At the voltage of $100 \mathrm{kV}$ the pulse of fast electrons current with duration of $0.2 \mathrm{~ns}$ and peak value of $400 \mathrm{~A}$ was obtained (Fig. 3). At the voltage of $200 \mathrm{kV}$ (Fig. 4) the peak value of fast electrons current pulse increased up to $1500 \mathrm{~A}$ at the same duration. Such sharp increasing was caused by increasing the efficiency of fast electrons generation with growth.

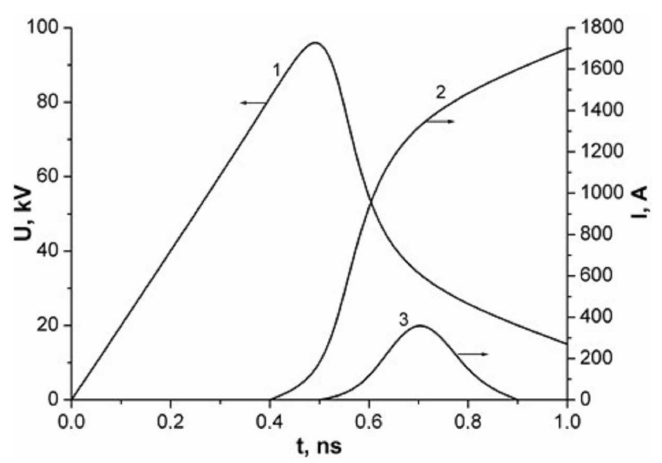

Fig. 3. Calculated oscillograms of voltage on the gap (curve 1), currents of the discharge (slow electrons) (2) and fast electrons (3). The gap was $1 \mathrm{~cm}$, peak voltage was $100 \mathrm{kV}$.

However, the growth of applying voltage required the decrease of the rise voltage front $\left(\tau_{\mathrm{f}}\right)$. For $100 \mathrm{kV}$ $\tau_{\mathrm{f}}^{\max }=0.5 \mathrm{~ns}$, for $200 \mathrm{kV} \tau_{\mathrm{f}}^{\max }=0.3 \mathrm{~ns}$. If $\tau_{\mathrm{f}}>\tau_{\mathrm{f}}^{\max }$, it was impossible to apply the required voltage because of breakdown the gap.

Another important characteristic of fast electron beam is an energy distribution function.

The set of energy distribution functions presented in Fig. 5 was calculated for various voltages on the gap. It 


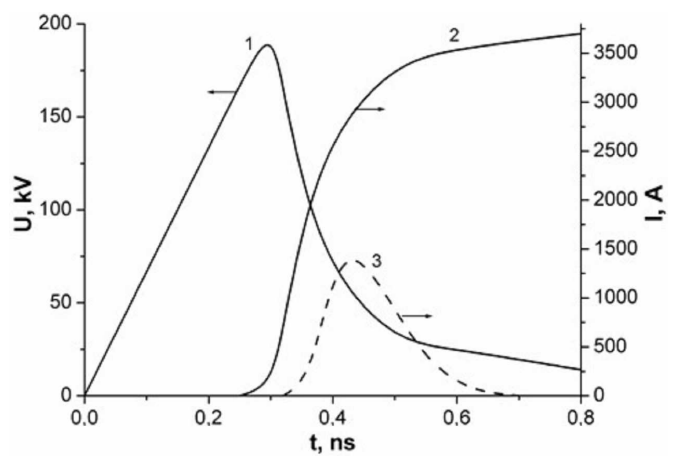

Fig. 4. Calculated oscillograms for peak voltage $200 \mathrm{kV}$. Notifications of curves are the same as in Fig. 3.

is visible that if the voltage grows with increase of the gap, the peak field strength in a gap has a stationary value $(100 \mathrm{kV} / \mathrm{cm})$, and growth of medial energy fast electrons occurs inefficiently. At stage-by-stage increase of the voltage on $25 \mathrm{kV}(75 \mathrm{kV}, 100 \mathrm{kV}, 125 \mathrm{kV})$ the maximum of energy distribution function was $32 \mathrm{keV}$ (curve 1), $46 \mathrm{keV}$ (curve 2), $55 \mathrm{keV}$ (curve 3) accordingly. It means that the growth of energy of fast electrons decreased from $14 \mathrm{keV}$ to $9 \mathrm{keV}$. More effective grows of fast electron energy provided by increasing the field strength in the gap. Therefore for example the magnification of this parameter twice (from 100 up to $200 \mathrm{kV} / \mathrm{cm}$ ) increased twice the energy of a maximum of a distribution function (46 keV, curve 2 and $92 \mathrm{keV}$, curve 4).

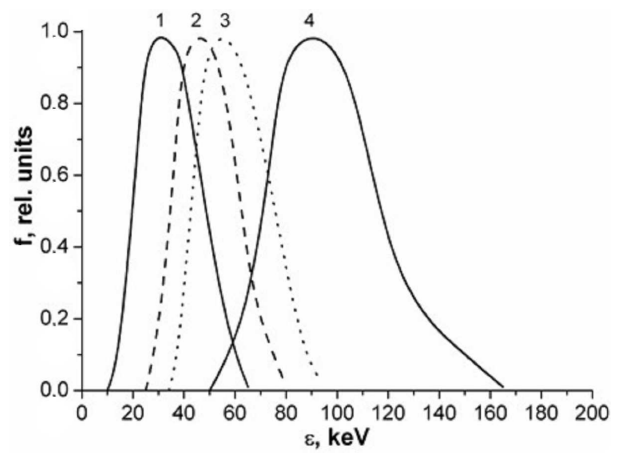

Fig. 5. Fast electron energy distributions. Curves 1, 2,3 correspond to voltage $75,100,125 \mathrm{kV}$ and to gap $0.75,1.0,1.25 \mathrm{~cm}$. The curve 4 corresponds to voltage $200 \mathrm{kV}$ and to gap $1 \mathrm{~cm}$.

Nevertheless, a major factor decreasing efficiency of acceleration of electrons in the circuit with one voltage source (Fig. 2a) is a voltage drop on the gap owing to growth of the current. As a result, the majority of fast electrons have not enough time for acceleration in the discharge gap. Therefore, the average energy of electrons does not exceed the half of applied voltage.

Efficiency of fast electrons acceleration can be more increased by using the circuit with two high-voltage generators and two consecutive gaps (Fig. 2b). The first gap acts as generator of the pulse of fast electrons current with the same characteristics as in a case of circuit with one voltage generator (Fig. 2a). Passing through the mesh, the fast electrons continue their acceleration in quasi-constant electric field of $30 \mathrm{kV} / \mathrm{cm}$ in the second gap. Time of development of breakdown in such field considerably exceeds duration of the current pulse of fast electrons.

The set of energy distribution functions of electrons on the energies, calculated for such system, is presented in Fig. 6. As can be seen from this figure, the medial energy of electrons is in all cases approximately $2 / 3$ from an accelerating voltage. The increase of medial energy of fast electrons is proportional to increase of an accelerating voltage.

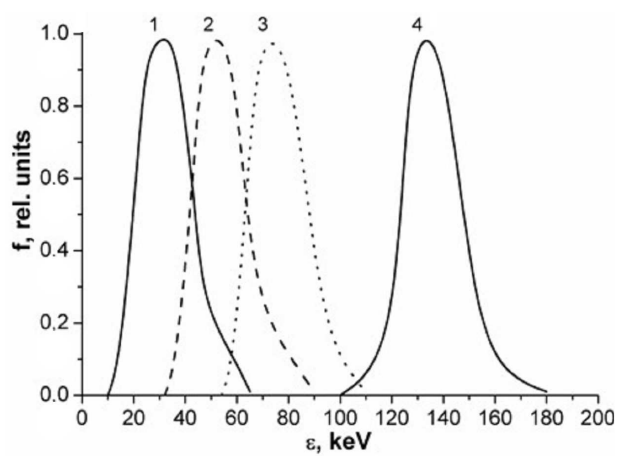

Fig. 6. Fast electron energy distributions. Notifications are the same as in Fig. 5.

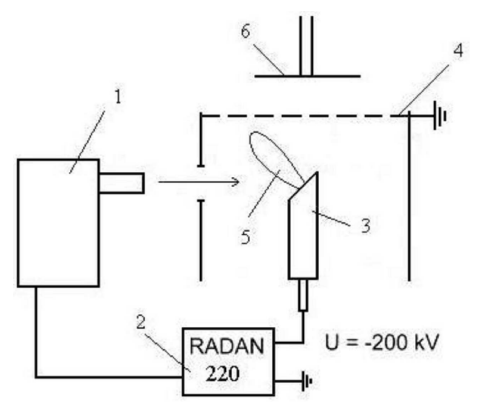

Fig. 7. The experimental setup. 1 - laser, 2 RADAN, 3 - graphite cathode, 4 - anode, 5 - laser plume, 6 - current shunt.

We tried to test experimentally the efficiency of suggested and theoretically proved method. The diagram of the experimental set up is given in Fig. 7. The laser (1) generated a pulse with duration of $1 \mathrm{~ms}$ and energy of $10 \mathrm{~J}$ which was focused by lens on surface of the graphite cathode (3). The diameter of cathode was $8 \mathrm{~mm}$. The distance between the cathode and anode was $18 \mathrm{~mm}$. The angle of the cathode edge was 45 and 20.5 degrees. It means that the angle between axis of plasma plume (5) and mesh anode (4) was accordingly 45 and 20.5 degrees. Therefore, unfortunately, only the small area of 
the plume could be used for generation of fast electrons.

With a help of pulse power system RADAN (2), the high voltage pulse with amplitude of $200 \mathrm{kV}$ and duration of 2 ns was applied to the gap with 2-10 ms time-delay after laser pulse. Such delay is necessary for finishing the expansion of laser plume.

The electron beam accelerated into the gap further fell on the current shunt (6), which was on $10 \mathrm{~mm}$ distance from the anode. The $10 \mathrm{~mm}$ distance excluded the falling of slow electrons on shunt. The diameter of shunt was $42 \mathrm{~mm}$. The voltage pulse on the shunt was measured by oscilloscope Tektronics TDS-360.

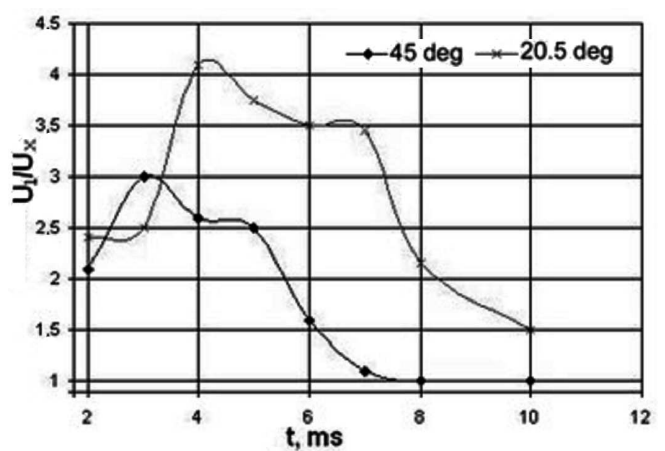

Fig. 8. Dependence $U_{1} / U_{x}$ on delay time.

Experiments showed that the amplitude of a voltage pulse on the shunt after laser impulse $U_{1}$ was essentially increased in comparison with amplitude in case of absence of laser pulse $U_{x}$. Dependence $U_{l} / U_{x}$ on delay time between laser pulse and high voltage pulse for edge corners of the cathode of 20.5 and 45 degrees are shown in Fig. 8. It is visible that there is an optimum delay time at which the fast electron current becomes maximum value.
Presence of delay time between peak value of fast electron current and laser pulse, and its dependence on angle of the cathode edge are caused, in our opinion, by dynamics of expansion of a laser plume.

We obtained the magnification of amplitude of a fast electron current more than in 4 times in comparison with absence of a laser impulse. According to our calculations the magnification of fast electron current should be much more than 4 times. This difference may be explained by angle between plasma plume axis and direction of fast electrons flow. Therefore, as we said above, only the small area of the plume was used for generation of fast electrons.

However, the experiment qualitatively confirms efficiency of suggested mechanism of fast electrons generation in air, and our calculations proved availability of its further research.

\section{Acknowledgments}

This research was supported by Russian Foundation for Basic Research, grants No. 08-02-00982-a, No. 08-0299065-r_ofi.

\section{References}

[1] L.P. Babich, T.V. Loko, V.A. Tsukerman, Sov. Phys. Usp. 33, 27 (1990).

[2] V.F. Tarasenko, S.I. Yakovlenko, Physics-Uspekhi 47, 887 (2004).

[3] G.A. Mesyats, S.D. Korovin, K.A. Sharypov, V.G. Shpak, S.A. Shunailov, M.I. Yalandin, Techn. Phys. Lett. 32, 18 (2006).

[4] V.V. Osipov, V.I. Solomonov, V.V. Platonov, O.A. Snigireva, M.G. Ivanov, V.V. Lisenkov, Quantum Electron. 35, 467 (2005). 